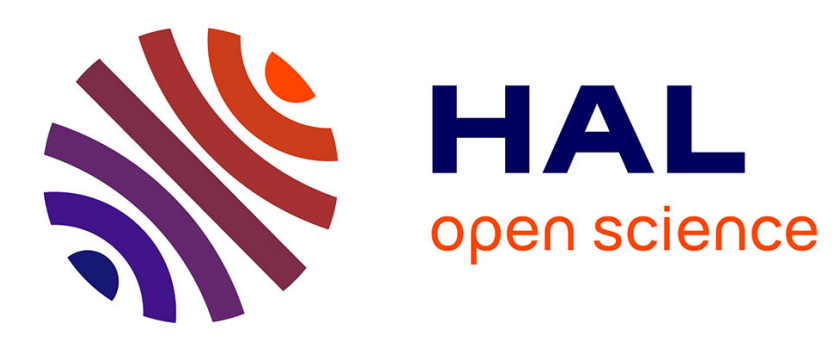

\title{
Chaos in variable stars: Topological analysis of W Vir model pulsations
}

Christophe Letellier, G. Gouesbet, F. Soufi, J.R. Buchler, Z. Kolláth

\section{To cite this version:}

Christophe Letellier, G. Gouesbet, F. Soufi, J.R. Buchler, Z. Kolláth. Chaos in variable stars: Topological analysis of W Vir model pulsations. Chaos: An Interdisciplinary Journal of Nonlinear Science, 1996, 6 (3), pp.466-476. 10.1063/1.166189 . hal-01596864

\section{HAL Id: hal-01596864 https://hal.science/hal-01596864}

Submitted on 18 May 2021

HAL is a multi-disciplinary open access archive for the deposit and dissemination of scientific research documents, whether they are published or not. The documents may come from teaching and research institutions in France or abroad, or from public or private research centers.
L'archive ouverte pluridisciplinaire HAL, est destinée au dépôt et à la diffusion de documents scientifiques de niveau recherche, publiés ou non, émanant des établissements d'enseignement et de recherche français ou étrangers, des laboratoires publics ou privés. 


\title{
Chaos in variable stars: Topological analysis of W Vir model pulsations
}

\author{
C. Letellier and G. Gouesbet \\ LESP, URA CNRS 230, INSA de Rouen, BP 08, 76130 Mont-Saint-Aignan, France \\ F. Soufi \\ DASGAL, Observatoire de Paris, Place Jules Jansen, 92190 Meudon, France \\ J. R. Buchler and Z. Kolláth \\ Physics Department, University of Florida, Gainesville, Florida 32611
}

(Received 19 December 1995; accepted for publication 26 June 1996)

The topological characterization of chaos is applied to the irregular pulsations of a model for a star of the W Virginis type, computed with a state-of-the-art numerical hydrodynamical code. The banded W Vir attractor is found to possess an additional twist when compared to the Rössler band. It is shown that the stellar light-curve contains the same dynamical information about the attractor as the stellar radius or as the radial velocity variations. (C) 1996 American Institute of Physics. [S1054-1500(96)01303-1]

From a chaotic time series generated by a lowdimensional dynamical system one can extract the lowest unstable periodic orbits. The relative topological organization (twisting) of these orbits can be exploited to characterize the dynamical attractor. In the present paper we explore the usefulness of this topological method for problems in astronomy by analyzing first as a sample problem the well-known Rössler dynamical system and then as a more realistic example numerical data from a hydrodynamical model of an irregularly pulsating star. Our ultimate purpose is the application to the light curves and radial velocity curves of variable star data.

\section{INTRODUCTION}

Observations and theory have shown that in classical variable stars such as the Cepheids and RR Lyræ stars, the pulsations are often periodic, to good accuracy. When they are multiperiodic they just involve a couple of frequencies. In contrast, the pulsations of many of their more luminous and metal poor Population II cousins are irregular with pulsation pseudo-periods larger than 15 days. In the last decade, an extensive hydrodynamical survey of models of $\mathrm{W}$ Virginis type stars with numerical codes ${ }^{1,2}$ has shown that the pulsations of the Population II objects are in fact chaotic in the dynamical sense of the word. The dominant clue pointing to the chaotic nature of these pulsations comes from the fact that, as a control parameter is varied, namely the equilibrium effective temperature of the models, the pulsations display a period-doubling cascade that is a route to chaotic dynamics (for a review, see Ref. 3).

In this paper, we study the irregular pulsations of a $\mathrm{W}$ Vir model computed with a state-of-the-art hydrodynamical code. Such a code provides the time series of three different variables, namely the stellar radius, the radial velocity and the luminosity. Our effort is devoted to the topological characterization of the different representations of the attractor as given by each of these three variables. Indeed, by using a reconstruction method introduced by Packard et al., ${ }^{4}$ one may reconstruct from a time series a state space equivalent to the original state space. In the case when the underlying temporal behavior is chaotic, a reconstructed chaotic attractor is then obtained.

The paper is organized as follows. In section II, we introduce the topological characterization procedure on the example of the Rössler system. This section is essentially of a pedagogic character to introduce concepts to be used later. Section III provides a complete topological analysis of the attractor induced by the stellar radius time series. In section IV the equivalence between the three variables output by the code is discussed from a dynamical point of view, and section $\mathrm{V}$ presents a conclusion.

\section{TOPOLOGICAL CHARACTERIZATION}

In the last few years several works discussed the topological description of chaotic attractors. In particular the idea has arisen that an attractor can be described by the population of periodic orbits, their related symbolic dynamics and their linking numbers. ${ }^{5}$ In three dimensional cases, periodic orbits may be viewed as knots ${ }^{6}$ and, consequently, they are robust with respect to smooth parameter changes allowing the definition of topological invariants under isotopy (continuous deformation).

The topological approach is based on the organization of periodic orbits. We now present the basic concepts of topological characterization and symbolic dynamics. For the sake of simplicity, we use the well-known Rössler attractor as an example.

\section{A. Template}

The Rössler system ${ }^{7}$ is defined by the ODEs

$$
\begin{aligned}
& \dot{x}=-y-z, \\
& \dot{y}=x+a y, \\
& \dot{z}=b+z(x-c),
\end{aligned}
$$




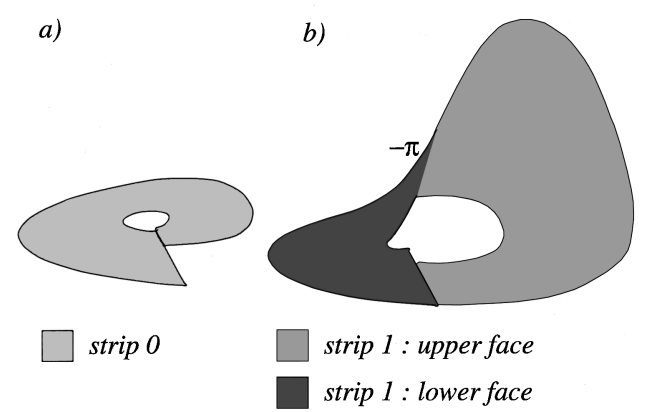

FIG. 1. The two strips of the Rössler attractor.

where $a, b, c$ are the control parameters. When $(a, b, c)=(0.398,2,4)$ the asymptotic motion settles down on to a strange chaotic attractor.

The attractor may be viewed as a simply stretched and folded band. Two different strips may be exhibited from this attractor (Fig. 1) (i) one which is located in the center of the attractor is a very simple strip without any $\pi$-twist [Fig. 1(a)] and (ii) a second strip which presents a negative $\pi$-twist [Fig. 1(b)] and is therefore similar to a Mœbius band. We may thus distinguish two topological regions on the attractor.

Following a pioneering paper by Birman and Williams, ${ }^{8}$ it has been shown ${ }^{5,6,9}$ that a template which encodes the topological properties of an attractor may be built in 3-dimensional state spaces. Such a template provides a view of the attractor that conveniently exhibits the different strips within the attractor and their relative organization. From the Rössler attractor, a template consisting of two strips is then extracted and displayed [Fig. 2(a)]. The band is split into two strips, one without any $\pi$-twist and one with a negative $\pi$-twist [Fig. 2(a)]. Following a standard insertion convention, ${ }^{9}$ strips must be reinjected into the bottom band from back to front, and from left to right. Consequently, a permutation between the strips is required, thereby leading to [Fig. 2(b)].

a)

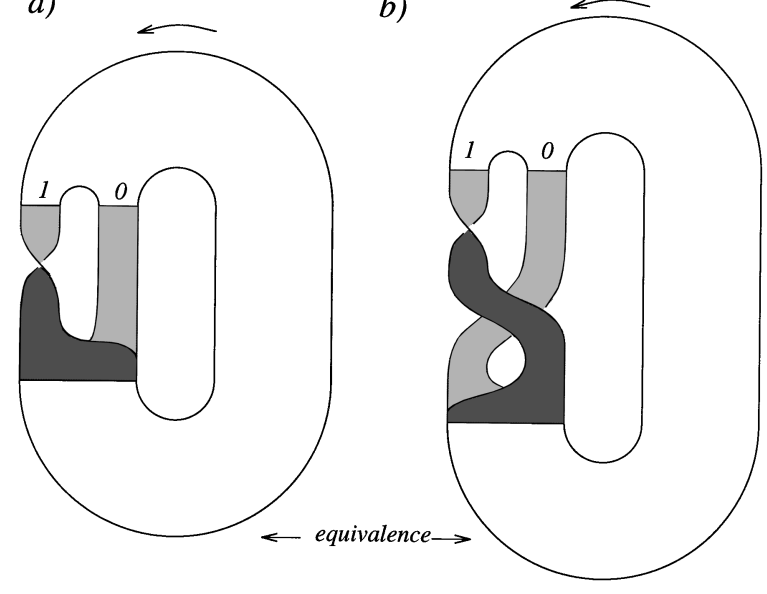

FIG. 2. Template of the Rössler attractor. A permutation between the strips is required by the standard insertion convention.
This convention allows an unambiguous description of the template by defining a linking matrix ${ }^{9}$ as follows. Diagonal elements $M(i, i)$ are equal to the number of $\pi$-twists number of the $i$ th strip and off-diagonal elements $M(i, j)$, $i \neq j$ are given by the algebraic number of intersections between the $i$ th and the $j$ th strips. One may then check that the Rössler template is defined by the linking matrix

$$
M \equiv\left(\begin{array}{cc}
0 & -1 \\
-1 & -1
\end{array}\right) .
$$

Each strip may be labelled: symbol 0 designs the simple strip while symbol 1 is associated with the strip which presents a negative $\pi$-twist. By this way, trajectories are encoded by a string of " 0 " and " 1. "' In particular, periodic orbits may be encoded in a one-to-one way. We have thus defined a symbolic dynamics. This procedure requires a precise partition of the attractor which is given by a first-return map to a Poincaré section.

\section{B. First-return map}

A Poincare section is defined as the set of intersections of a chaotic trajectory with a plane transverse to the flow. For the Rössler system, a suitable Poincare section is given by

$$
P \equiv\left\{(y, z) \in \mathbb{R}^{2} \mid x=x_{-}, \dot{x}>0\right\},
$$

where $x_{-}=\left(c-\sqrt{c^{2}-4 a b}\right) / 2$ is the $x$-coordinate of the central fixed point. ${ }^{10}$

The first-return map is computed with the $y$-variable. It presents two monotonic branches : an increasing branch associated with strip 0 and a decreasing branch associated with strip 1. The critical point $y_{c}$ which separates the branches precisely defines the partition. In our case, $y_{c}=-3.04$. Thus, each intersection $y_{i}$ with the Poincare plane corresponds to a code $K\left(y_{i}\right)$ given by

$$
K\left(y_{i}\right)=\left\{\begin{array}{lll}
0 & \text { if } & y_{i}>y_{c}, \\
1 & \text { if } & y_{i}<y_{c} .
\end{array}\right.
$$

Once periodic orbits are extracted (in the present work by using a Newton-Raphson iteration scheme), periodic points in the Poincare section may be encoded. An orbit of period $p$ has $p$ periodic points and is represented by a string $S$ of $p$-codes

$$
S=K\left(y_{1}\right) K\left(y_{2}\right) \ldots K\left(y_{p}\right),
$$

where $y_{i}$ 's are the $y$-coordinates of the periodic points.

\section{Unimodal order}

Each period- $p$ point is represented by a symbolic sequence of $p$ symbols. The $i$ th point of a period- $p$ orbit is labelled by the string

$$
S_{i}=K\left(y_{i}\right) K\left(y_{i+1}\right) \ldots K\left(y_{p}\right) K\left(y_{1}\right) \ldots K\left(y_{i-1}\right) .
$$

All periodic points are then ordered by the unimodal order. ${ }^{11,12}$

Definition 1. The unimodal order $<_{1}$ on the symbol set 0,1 is defined as follows. 
Let us consider two symbolic sequences

$$
W_{1}=\sigma_{1} \sigma_{2} \ldots \sigma_{k} \sigma_{k+1} \ldots
$$

and

$$
W_{2}=\tau_{1} \tau_{2} \ldots \tau_{k} \tau_{k+1} \ldots,
$$

where $\sigma_{i}$ 's and $\tau_{j}$ 's designate the codes. Suppose $\sigma_{i}=\tau_{i}$ for all $i<k$ and $\sigma_{k} \neq \tau_{k}$. Let $W^{*}=\sigma_{1} \ldots \sigma_{k-1}=\tau_{1} \ldots \tau_{k-1}$ be the common part between $W_{1}$ and $W_{2}$. We say that a string $\sigma_{1} \sigma_{2} \ldots \sigma_{k-1}$ is even (odd) if the sum $\sum_{i=1}^{k-1} \sigma_{i}$ is even (odd), and that $W^{*}$ is even when no common part is found between $W_{1}$ and $W_{2}$. Then, we have

$$
\left\{\begin{array}{llllll}
W_{1}<{ }_{1} W_{2} & \text { if } & W^{*} & \text { is even } & \text { and } & \sigma_{k}<\tau_{k}, \\
W_{1}<{ }_{1} W_{2} & \text { if } & W^{*} & \text { is odd } & \text { and } & \tau_{k}<\sigma_{k}, \\
W_{2}<{ }_{1} W_{1} & \text { if } & W^{*} & \text { is odd } & \text { and } & \sigma_{k}<\tau_{k}, \\
W_{2}<{ }_{1} W_{1} & \text { if } & W^{*} & \text { is even } & \text { and } & \tau_{k}<\sigma_{k} .
\end{array}\right.
$$

When $W_{1}<{ }_{1} W_{2}$, we say that $W_{2}$ implies $W_{1}$.

A period- $p$ orbit will be denoted by the symbolic sequence $W_{i}$ which implies the $(p-1)$ others. This sequence is noted $\left(W_{i}\right)$, with parentheses, and is called the orbital sequence. Two orbital sequences may also be ordered following the unimodal order. When the orbital sequence $\left(W_{2}\right)$ implies the orbital sequence $\left(W_{1}\right)$, we say that $\left(W_{2}\right)$ forces $\left(W_{1}\right)$ and we note $\left(W_{1}\right)<_{2}\left(W_{2}\right)$ where $<_{2}$ is the forcing order. By this way, all periodic orbits are ordered. The orbital sequence which forces all orbital sequences extracted from the attractor is called the kneading sequence. Within the Rössler attractor, the kneading sequence (among the orbits of period less than 12) is found to be (10111101010). ${ }^{10}$ All orbits forced by the kneading sequence are found to be present in the attractor up to period 11 included, at least.

\section{Symbolic plane}

With numerical systems, an orbit spectrum is always well-known, but within the limits imposed by round-off errors (since orbits are extracted by integrating the vector field). Nevertheless, in the case of experimental data, periodic orbits are extracted from a time series by using a closereturn method in a reconstructed phase space. Due to the limited amount of data and to the influence of external noise, the orbit spectrum is rarely well-known. In particular, as shown by Tufillaro et al., ${ }^{13}$ the population of periodic orbits crucially depends on the length of the time series. Consequently, the determination of the kneading sequence is rather inaccurate when using short experimental time series.

Fang ${ }^{14}$ has shown that an empirical procedure (also used by Tufillaro et al. ${ }^{13}$ ) may however exhibit the pruning front introduced by Cvitanovic ${ }^{15}$ and, consequently, the kneading sequence. To explain this procedure, let us first recall that a chaotic trajectory forms a string

$$
\mathbf{S}=\ldots \sigma_{-3} \sigma_{-2} \sigma_{-1} \sigma_{0} \sigma_{1} \sigma_{2} \sigma_{3} \ldots,
$$

where $\sigma_{0}$ is the present, $\sigma_{-i}$ 's the past and $\sigma_{i}$ 's the future $(i>0)$.

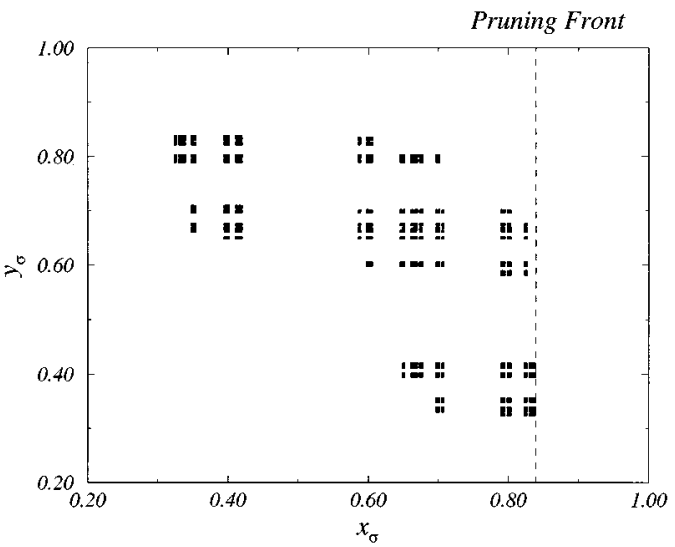

FIG. 3. Symbolic plane of the Rössler attractor: The orbit spectrum is governed by the unimodal order as shown by the pruning front well-estimated by a line.

Symbolic coordinates which span a symbolic plane are then defined on the future and the past as follows:

$$
\begin{aligned}
& x_{\sigma}(s)=\sum_{i=1}^{D} \frac{b_{i}}{2^{i}} \quad \text { where } \quad b_{i}=\sum_{j=1}^{i} \sigma_{j} \quad(\bmod 2) \text {, } \\
& y_{\sigma}(s)=\sum_{i=1}^{D} \frac{c_{i}}{2^{i}} \quad \text { where } \quad c_{i}=\sum_{j=0}^{i-1} \sigma_{-j} \quad(\bmod 2),
\end{aligned}
$$

where

$\mathbf{S}=\sigma_{-D} \ldots \sigma_{-3} \sigma_{-2} \sigma_{-1} \sigma_{0} \sigma_{1} \sigma_{2} \sigma_{3} \ldots \sigma_{D}$.

If $s$ is an infinite symbol string generated by a chaotic orbit, then $D$ is infinity in the above definition. However, since we are dealing with finite data sets, Tufillaro et al. ${ }^{13}$ approximate the symbolic plane coordinates of a point by taking $D=16$. In this way, we can use a finite symbol string from a chaotic trajectory to generate a sequence of points on the symbolic plane displayed. The symbolic plane for the Rössler attractor is given in Fig. 3. In the present case of an orbit spectrum governed by the unimodal order, the pruning front is suitably estimated by a line. ${ }^{14,16}$

The symbolic coordinate $x_{\sigma}$ of the pruning front allows us to determine the kneading sequence. Indeed, after having computed the orbital sequences of periodic orbits, the kneading sequence is associated with the orbital sequence whose $x_{\sigma}$ is closest to the pruning front.

In the Rössler case, the pruning front is located at $x_{\sigma}=0.8376$. From the orbit spectrum of the Rössler attractor, the kneading sequence is found to be (10111101010) whose symbolic coordinate is 0.8375 in good agreement with the pruning front location. In the case of experimental data, the symbolic plane will be systematically used to check the orbit spectrum.

\section{E. Template validation}

A template of the Rössler attractor has been given in section II A and the orbit spectrum is extracted. The template must now be checked by comparing linking numbers predicted by the template and the ones counted on the attractor. 


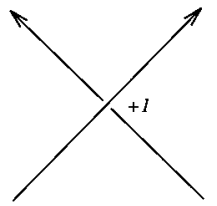

(a)

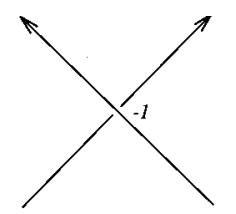

(b)
FIG. 4. Crossing convention: (a) positive crossing and (b) negative crossing.

The linking number $L\left(N_{1}, N_{2}\right)$ of an orbit pair is given by the half-sum of the oriented crossings (following the convention, given in Fig. 4, due to Melvin and Tufillaro ${ }^{9}$ ) on a regular plane projection of orbits $N_{1}$ and $N_{2}$. For example, linking number $L(1011,1)$ is equal to -2 (Fig. 5).

A comparison with the linking number $L(1011,1)$ predicted from the template is achieved by using an algebraic relation between symbolic dynamics and linking matrix according to ${ }^{17}$

$$
L\left(N_{1}, N_{2}\right)=\frac{1}{2}\left[\sum_{i=1}^{p_{1}} \sum_{j=1}^{p_{2}} M\left(\sigma_{i}, \sigma_{j}\right)+N_{\text {lay }}\left(N_{1}, N_{2}\right)\right],
$$

where $N_{1}$ and $N_{2}$ are two orbits of period $p_{1}$ and $p_{2}$, respectively. Also, $M\left(\sigma_{i}, \sigma_{j}\right)$ are the linking matrix elements and $N_{\text {lay }}\left(N_{1}, N_{2}\right)$ is the layering number determined by using a layering graph (sketched in Fig. 6 for the couple $(1011,1)$, see Ref. 17 for details).

In the present case, we obtain

$$
\begin{aligned}
L(1011,1) & =\frac{1}{2}\left[3 M(1,1)+M(1,0)+N_{\text {lay }}(1011,1)\right] \\
& =\frac{1}{2}[-3-1+0]=-2,
\end{aligned}
$$

i.e., the linking number $L(1011,1)$ predicted from the template is the same as on the attractor. The template is therefore compatible with the attractor. For utmost rigor, a few linking numbers are needed to completely check the template.

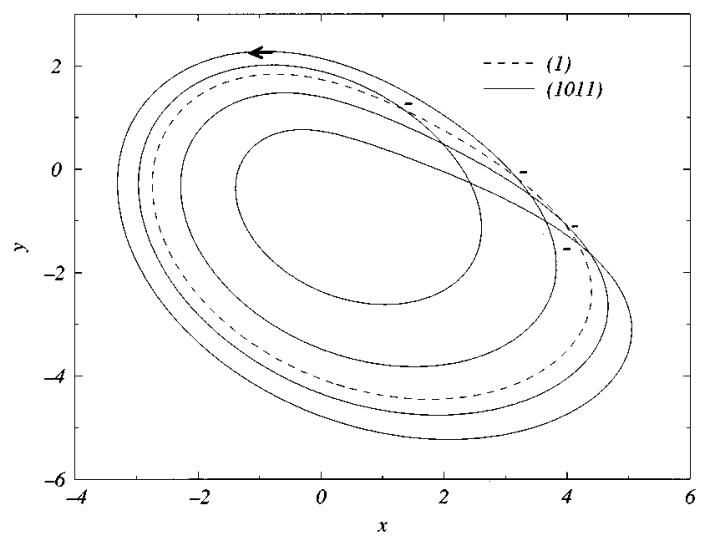

FIG. 5. Plane projection of the orbit couple $(1011,1)$. The linking number $L(1011,1)=\frac{1}{2}[-4]=-2$. Crossings are signed by inspection on the third coordinate.

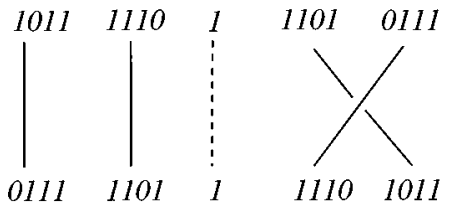

FIG. 6. Layering graph between (1) and (1011). Lower base is given by the unimodal order of periodic points. Upper base is obtained by using a reverse permutation of periodic points of strip 1 (whose symbolic sequences begin by a " 1 ") since strip 1 has a odd number of $\pi$-twists. Periodic points of strip 1 are thereafter permuted (again by using a reverse permutation) with periodic points of strip 0 since the intersection number $M(1,0)$ between strip 1 and strip 0 is odd. The layering number $N_{\text {lay }}(1011,1)$ is equal to the sum of the intersections between (1011) and (1) (self-intersections are not taken into account). Here $N_{\text {lay }}(1011,1)$ is null.

As the template which carries the periodic orbits is identified, the organization of the orbits within the attractor is known. For a complete discussion about equivalence between periodic orbits embedded within a strange attractor and orbits of the template, see Ref. 18.

From a practical point of view, depending on the complexity of the dynamics, the number of points per cycle may be taken from 20 in the case of very simple dynamics like the simple Rössler band up to 200 or more when the dynamics requires many symbols for its description. In other words, the amount of data available determines the knowledge of the orbit spectrum which may be gained, high period orbits requiring more data. For low period orbits which are usually sufficient to specify the orbit spectrum with a reasonable accuracy, 50 to a few hundred characteristic cycles, with as few as 20 data per cycle, may be sufficient for an accurate enough characterization of the attractor, making the theorist requirements compatible with limited experimental observations. The acceptable noise level depends on the structure of the attractor, i.e., whether it possesses a large number of strips with many $\pi$-twists. Clearly a lower noise level is required with more strips.

\section{W VIR ATTRACTOR ANALYSIS}

\section{A. The model}

The W Vir model considered here, namely model D5200 of Ref. 2, is a realistic and state-of-the-art model in so far as it contains all the complications that arise both in the equations of state and in the opacity due to partial $\mathrm{H}$ and $\mathrm{He}$ ionizations. The star has a mass of $0.6 M_{\odot}$, a luminosity of $500 L_{\odot}$, a hydrogen abundance $X=0.7$, and a heavy element abundance $Z=0.005$. This particular model is chosen because it generates quite irregular pulsations, exhibiting an almost fully developed chaos whose behavior appears, at least superficially, to be similar to the Rössler band behavior.

Three time series are provided by integrating the hydrodynamical code, namely the stellar radius $R(t)$, the radial surface velocity $v_{R}(t)$ and the luminosity $L(t)$. These variables are displayed in Fig. 7, with a sampling time $\delta t=210^{4} s$, i.e., $\approx 0.02 \times$ the "period" or cycling time.

A global flow reconstruction from the radius time series by Serre et al. ${ }^{19}$ showed that the chaotic attractor is not only 


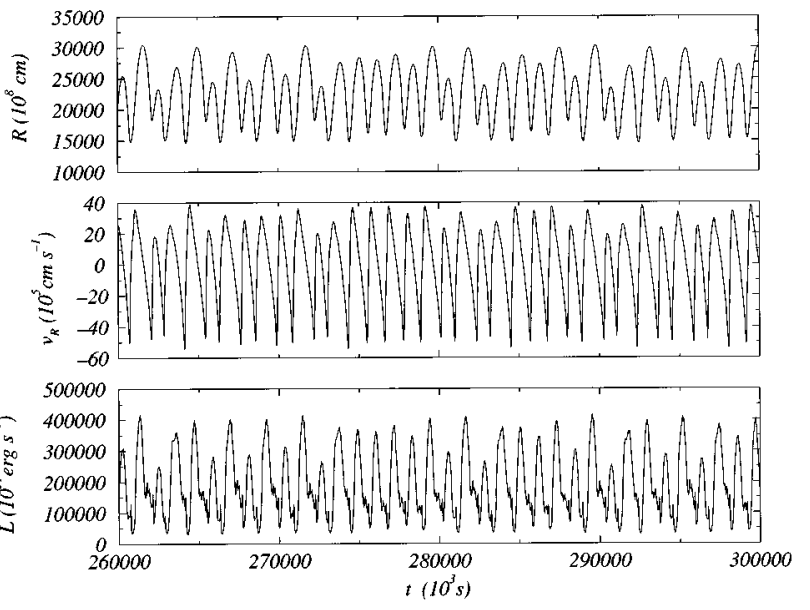

FIG. 7. Time series of the radius $R$, the radial velocity $v_{R}$ and the luminosity $L$ of the star.

embeddable in 3D, but that it also has a global physical dimension of 3. Furthermore from a computation of the Lyapunov exponents they showed that the chaotic pulsational behavior is characterized by a fractal (Lyapunov) dimension equal to about 2.02. They embedded the attractor in a 3D space spanned by delay coordinates ${ }^{20}$

$$
\{R(t), R(t+\tau), R(t+2 \tau)\},
$$

where $\tau=15 \delta t$ is the time delay. The corresponding $R$-induced attractor is displayed in Fig. 8 .

On the projection of the attractor (Fig. 8), it appears that the dynamics of the W Vir attractor is rather weakly dissipative when compared to the Rössler dynamics. Such a weakly dissipative behavior is clearly exhibited in Poincaré sections, as displayed in Fig. 9.

In weakly dissipative cases, Grassberger et al. ${ }^{21}$ showed that a good partition should pass through primary tangencies between stable and unstable manifolds. This is a generalization of the fact that the generating partition for the logistic map passes through the critical point. The kneading sequence which forces all sequences of periodic orbits present within the attractor is therefore replaced by a set of kneading se-

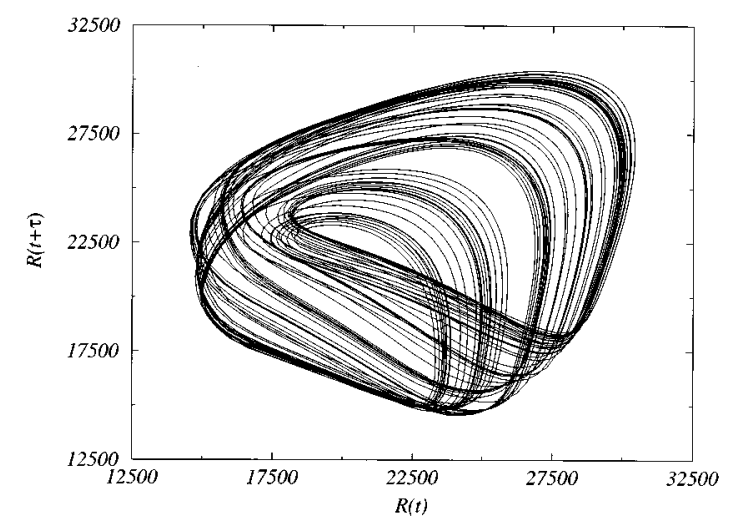

FIG. 8. Projection of the attractor in the plane defined by $R(t), R(t+\tau)$.
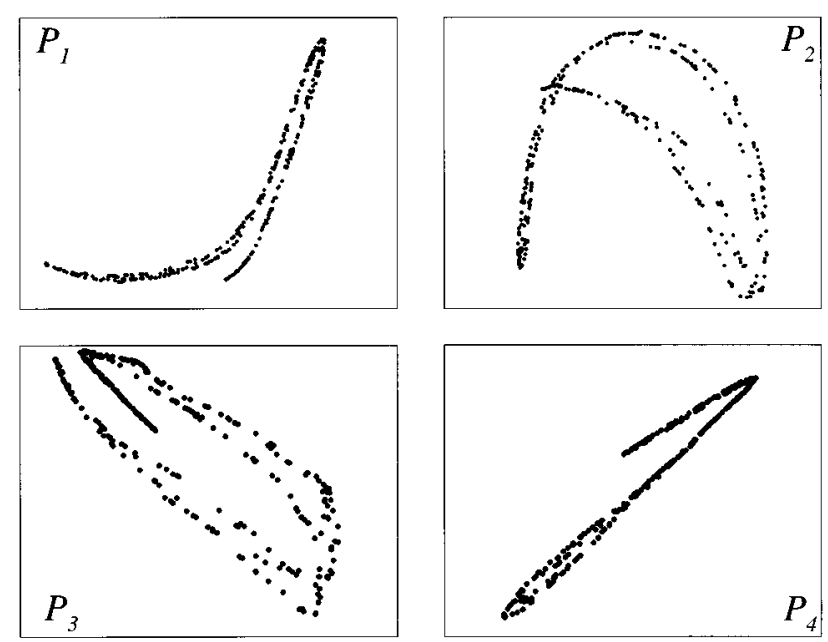

FIG. 9. Four different Poincare sections of the $R$-induced attractor which show the nontrivial structure of the stretched and folded band.

quences corresponding to orbits starting at primary tangencies. Such an analysis has recently been provided by Fang in the case of the Hénon map by using a symbolic plane to exhibit the set of kneading sequences. ${ }^{14}$ For instance, such a partition is displayed on Fig. 10 for the Hénon map with $a=1.4$ and $b=0.3$. One may remark that the partition is not necessarily given by the maxima of the layered structure.

Unfortunately, the extraction of primary tangencies requires the knowledge of the equations governing the Poincaré map of the dynamical system. Consequently, we cannot easily determine a perfect partition of the $R$-induced attractor, and periodic orbits in principle cannot safely be encoded. Moreover, the first-return map to the Poincare section $P_{R}$ defined by

$$
P_{R} \equiv\left\{R(t), R(t+\tau) \in \mathbb{R}^{2} \mid R(t)=R(t+\tau), \dot{R}>0\right\}
$$

exhibits a layered structure (Fig. 11) which is a signature of a weakly dissipative dynamics when compared to Rössler. Nevertheless, two branches may be clearly approximately exhibited and a single critical point $R_{c}$ may be defined at

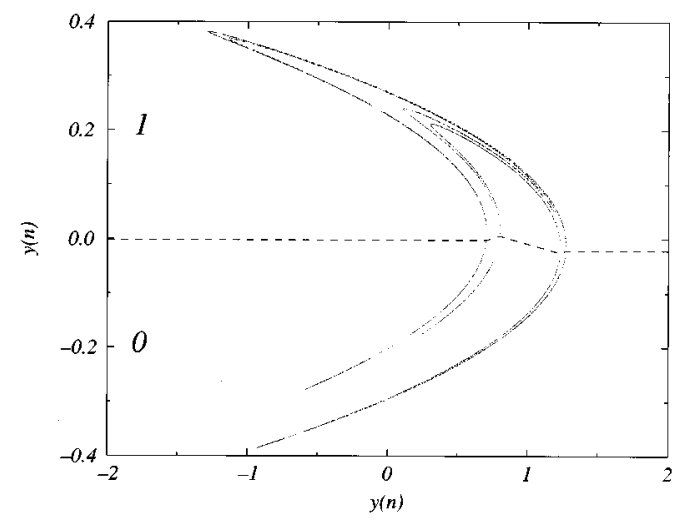

FIG. 10. Partition of the Hénon map by the primary tangencies between stable and unstable manifolds $((a, b)=(1.4,0.3))$. 


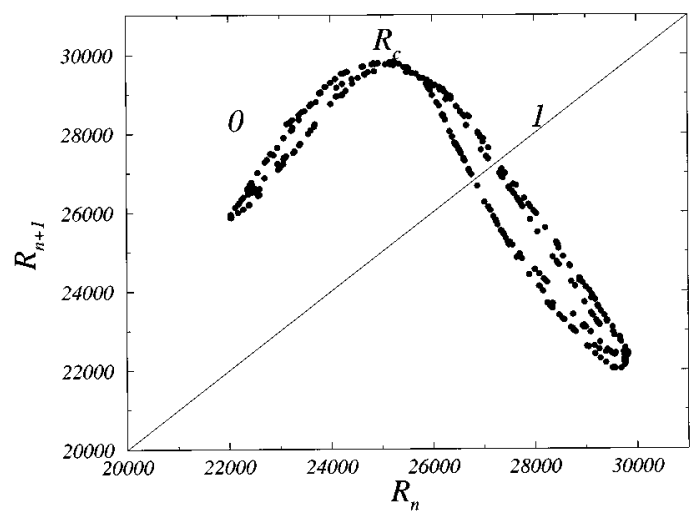

FIG. 11. First return map to the Poincare section $P_{R}$ of the $R$-induced attractor.

$R_{c}=24900$. Indeed, Fang has shown that symbolic sequences with small period can be described by the unimodal order in many systems whose dissipation rate is not too large. ${ }^{15,22}$

According to this map, a template consisting of two strips could possibly synthesize the $R$-induced attractor of the W Vir model. Examining the Poincaré sections of Fig. 9 which provide other points of view on the attractor, it appears that the use of two strips to propose a template can only be an approximation. Furthermore, the maps are not unidimensional. We shall, however, see that a two-strip approximate template will nevertheless provide pertinent information, valid for periodic orbits of small periods.

We first define a partition based on the first-return map displayed in Fig. 11. Periodic orbits are hereafter extracted by using a close recurrence technique ${ }^{23}$ and encoded by a symbolic dynamics with codes

$$
\begin{aligned}
& 0 \quad \text { if } R<R_{c}, \\
& 1 \quad \text { if } R>R_{c} .
\end{aligned}
$$

The population of periodic orbits is reported in Table I. Let us note, however, that the number of points in the Poin-

TABLE I. Population of periodic orbits within the $R$-induced attractor $A_{R}$ and the $L$-induced attractor $A_{L}$. When a periodic orbit is found, the close

\begin{tabular}{|c|c|c|c|}
\hline Period & (W) & $\begin{array}{l}A_{R} \\
\boldsymbol{\epsilon} \%\end{array}$ & $\begin{array}{l}A_{L} \\
\epsilon \%\end{array}$ \\
\hline 1 & 1 & 3.3 & 1.3 \\
\hline 2 & 10 & 2.3 & 0.3 \\
\hline \multicolumn{4}{|l|}{3} \\
\hline 4 & 1011 & 0.4 & 1.1 \\
\hline \multirow[t]{2}{*}{5} & 10111 & 4.0 & 1.7 \\
\hline & 10110 & 1.1 & 1.8 \\
\hline \multirow[t]{2}{*}{6} & 101110 & 0.1 & 0.3 \\
\hline & 101111 & 2.4 & 3.3 \\
\hline \multirow[t]{2}{*}{7} & 1011111 & 0.1 & 3.0 \\
\hline & 1011110 & 0.1 & 0.1 \\
\hline \multirow[t]{3}{*}{8} & 10111010 & 1.0 & 1.0 \\
\hline & 10111110 & & \\
\hline & 10111111 & 0.4 & 0.2 \\
\hline
\end{tabular}
return distance $\epsilon$ is reported (in $\%$ of the attractor size).

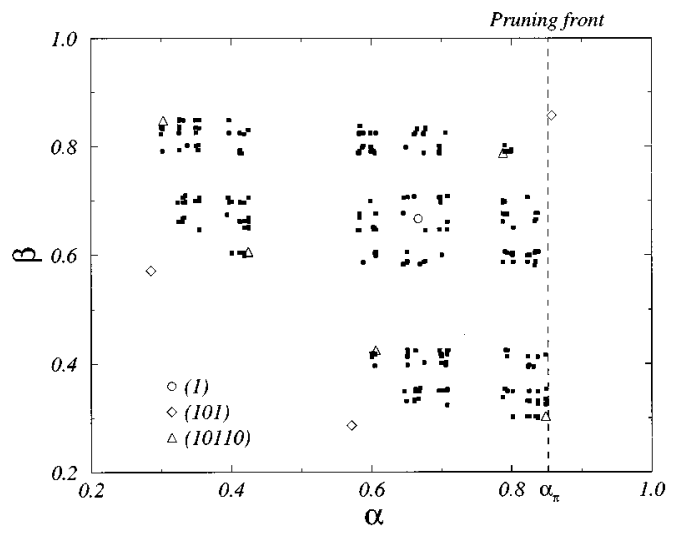

FIG. 12. Symbolic plane of the $R$-induced attractor. A few periodic orbits are reported. The pruning front may be approximated by the dashed line. One may remark that the neighborhood of the orbit encoded by (1) is not well visited by the trajectory. This fact explains the relatively large distance of close return for this period-1 orbit.

caré section is too small to provide a very accurate knowledge of the population of periodic orbits. For instance, the period-1 orbit encoded by (1) is found with a close return distance of $3.3 \%$. Such a distance is rather large for a period-1 orbit. Actually, due to the limited time series length and available initial conditions, the trajectory does not visit well the neighborhood of this orbit. In particular, for short time series, the probability of visiting the neighborhood of a given orbit sensitivity depends on the initial conditions. ${ }^{23}$

According to the unimodal forcing order, the kneading sequence of the $R$-induced attractor is given by $(\overline{10110})$. Thus, the saddle-node bifurcation which creates the pair of period-3 orbits encoded by (101) and (100) has not yet occurred, explaining why no period-3 orbit is found within the attractor (for more details, see Ref. 24). One may also remark that the period-8 orbit encoded by (10111110) is not found within the attractor although it is forced by the kneading sequence (Table I). This is not very surprising since the longer the periodic orbit, the lower the probability to detect it. $^{13,23}$ In order to check previous statements, the symbolic plane is computed and displayed in Fig. 12.

The pruning front is well approximated by a line located at $\alpha_{p}=0.8499$. As the leading symbolic coordinate $\alpha_{(10110)}=0.8485$, the kneading sequence is here confirmed. Moreover, as the leading symbolic coordinate $\alpha_{(101)}$ is equal to 0.8571 and, consequently, is greater than $\alpha_{p}$ (Fig. 12), period-3 orbits are not present within the attractor.

One may remark that the neighborhood along the line approximating the pruning front is not well visited for high $\beta$-values, a fact which could indicate the existence of a multimodal order. Nevertheless, due to the limited time series length, it is rather delicate to definitely state about the possible occurrence of a multimodal order to govern the population of periodic orbits. It is also true that the first-return map exhibits a layered structure but the amount of data is too small to resolve this issue. In practice, considering the results obtained, it is sufficient to assume that the dynamics is gov- 


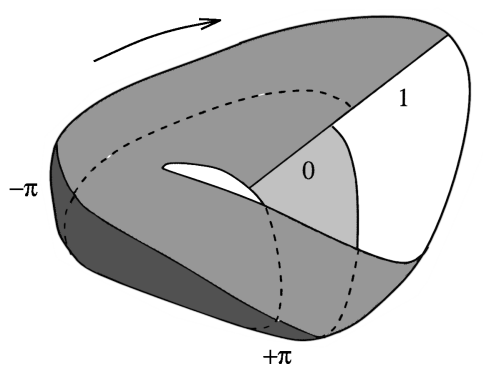

$\begin{array}{llll} & \square \text { upper face } & \square \text { upper face } \\ \text { Strip 1 } & \text { Strip 0 } & \\ & \square \text { lower face } & & \square\end{array}$

FIG. 13. Mask of the $R$-induced attractor. Two strips are exhibited. Strip 1 presents a negative $\pi$-twist and strip 0 undergoes successively through one positive and one negative $\pi$-twists.

erned by an unimodal forcing order. With this assumption which is clearly an approximation, we are able to characterize the attractor in a sufficient way. Furthermore, a more refined description would necessitate a deep understanding of topological characterization in the case of weakly dissipative systems. Such an understanding is still beyond reach.

\section{B. Template extraction}

In view of Fig. 11, we assume that the first-return map consists approximately of two monotonic branches. The $R$-induced attractor must therefore be divided into two different strips. The first, labelled 0 , with an even local torsion, is associated with the increasing branch, and the second, labelled 1, with an odd local torsion, is associated with the decreasing branch. By building an approximate mask of the attractor $A_{R}$, one can easily exhibit the two strips (Fig. 13). This mask is approximated in the sense that the $R$-induced attractor is reduced to a thin stretched and folded band, i.e., does not take into account the layered structures exhibited on the Poincaré sections displayed in Fig. 9.

Under a revolution on the attractor, strip 1 presents one positive $\pi$-twist while strip 0 undergoes successively one positive and one negative $\pi$-twist, i.e., has a zero local torsion. This mask may therefore be synthesized by a template which has one positive global torsion and two strips. One of which has a negative local $\pi$-twist (Fig. 14). The $R$-induced attractor has therefore an additional global $\pi$-twist when compared to the Rössler band. As displayed in Fig. 14, the global torsion may be reduced within the ribbon graph.

The template is defined by the following linking matrix:

$$
M=\left(\begin{array}{ll}
0 & 0 \\
0 & 1
\end{array}\right) \text {. }
$$

The template has now to be checked by comparing linking numbers predicted by the template with linking numbers computed on a plane projection. For instance, we obtain

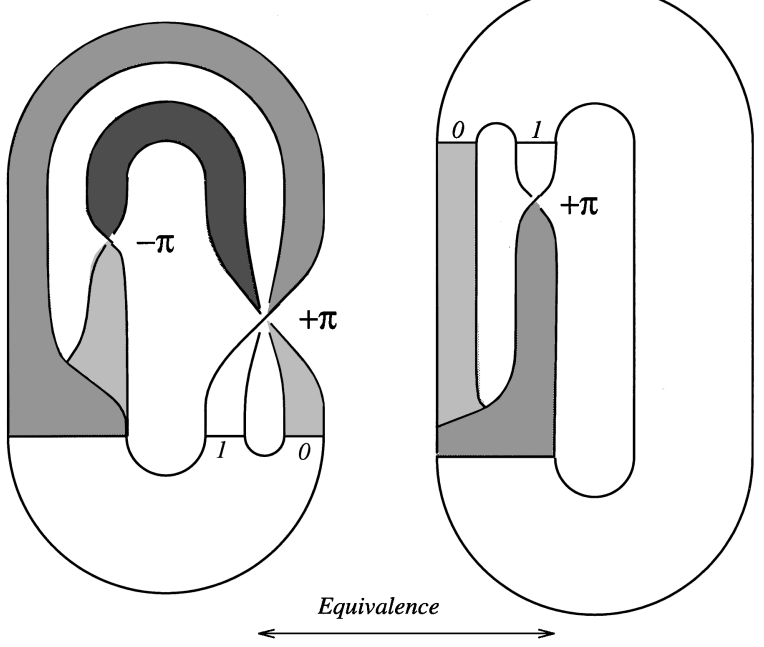

FIG. 14. Template of the $R$-induced attractor. The global torsion may be reduced within the ribbon graph. The proposed template is represented with respect to the standard insertion convention.

$L(1011,10)$

$$
\begin{aligned}
& =\frac{1}{2}\left[3 M(1,1)+4 M(1,0)+M\left(0,0+N_{\text {lay }}(1011,10)\right]\right. \\
& =\frac{1}{2}[+3+0+3]=+3
\end{aligned}
$$

and

$L(10111,10)$

$$
\begin{aligned}
& =\frac{1}{2}\left[4 M(1,1)+5 M(1,0)+M(0,0)+N_{\text {lay }}(10111,10)\right] \\
& =\frac{1}{2}[+4+0+0+4]=+4,
\end{aligned}
$$

where the layering numbers $N_{\text {lay }}(1011,10)$ and $N_{\text {lay }}(10111,10)$ are given by the layering graphs displayed in Fig. 15.

The pairs of periodic orbits are displayed on plane projections (Fig. 16) where oriented crossings are counted. The linking numbers $L(1011,10)$ and $L(10111,10)$ are found to be equal to +3 and +4 , respectively. Template predicted and counted linking numbers are therefore found to be equal. Linking numbers $L(10,1)$ and $L(1011,1)$ are also found to be equal to the template predictions. Nevertheless, by computing many oriented crossings for higher period orbits we have noted that a few of them are found with a sign opposite to the template prediction. Such reversed oriented crossings are a signature of the layered structure which is not taken into account by the template. Nevertheless, this layered structure has been found to be irrelevant on the relative organization of small period orbits (at least up to period 5), i.e., linking numbers of small period orbits are in agreement with the template predictions. Consequently, the proposed template is a good synthesis of the topology of the $R$-induced attractor. We also note that the topology of the attractor in 


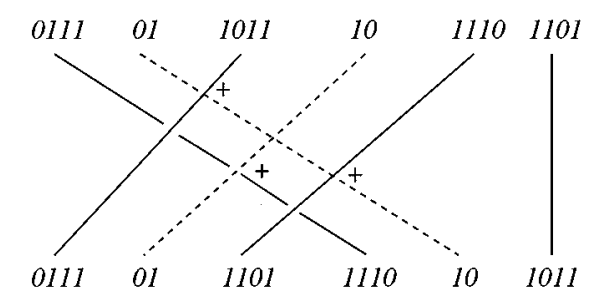

(a)

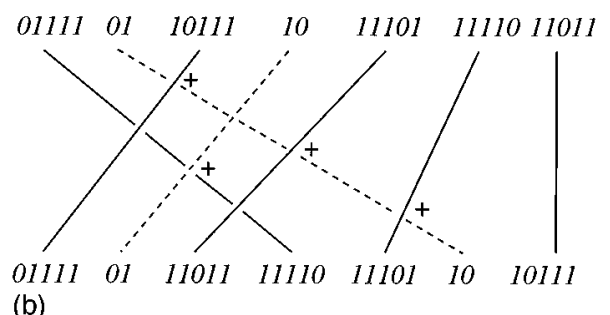

FIG. 15. The layering graphs of the pair $(1011,10)$ and $(10111,10)$. (a) $N_{\text {lay }}(1011,10)=+3$. (b) $N_{\text {lay }}(10111,10)=+4$.

the space spanned by the three coordinates $R, v_{R}$ and $L$ has been found to be the same as for the $R$-induced attractor.

\section{Equivalence between the different variables}

According to the embedding theorem ${ }^{25}$ it should be possible to use any physical variable for the reconstruction as long as it is generic and the embedding dimension large
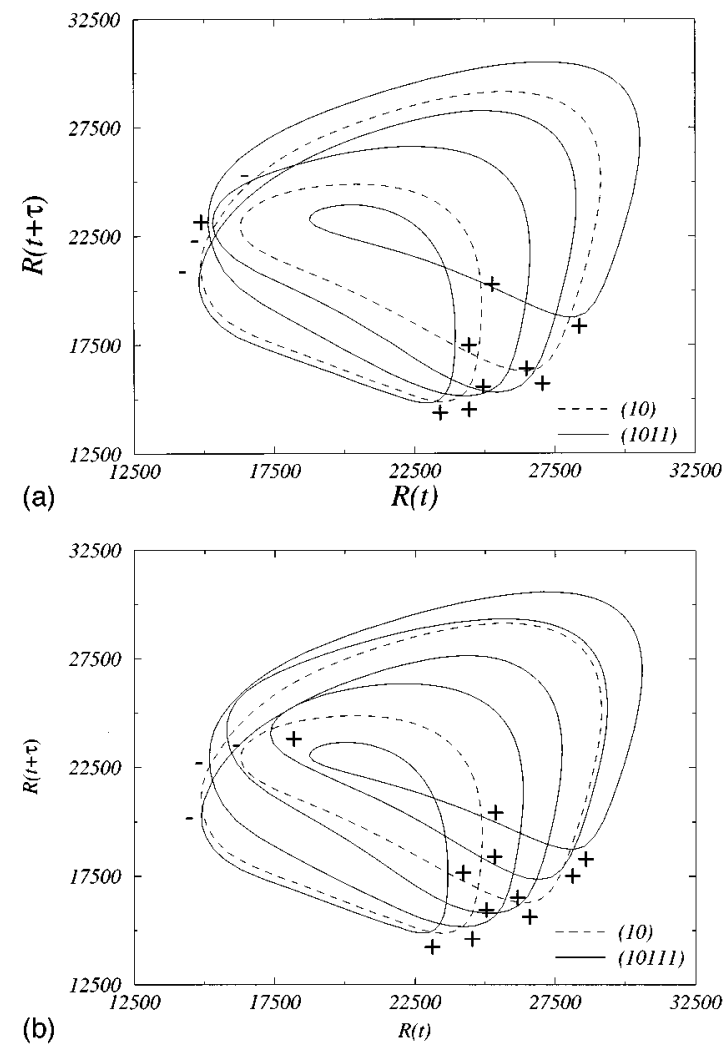

FIG. 16. Plane projection of two pairs of periodic orbits. (a) $L(1011,10)=\frac{1}{2}(-3+9)=+3$. (b) $L(10111,10)=\frac{1}{2}(-3+11)=+4$.

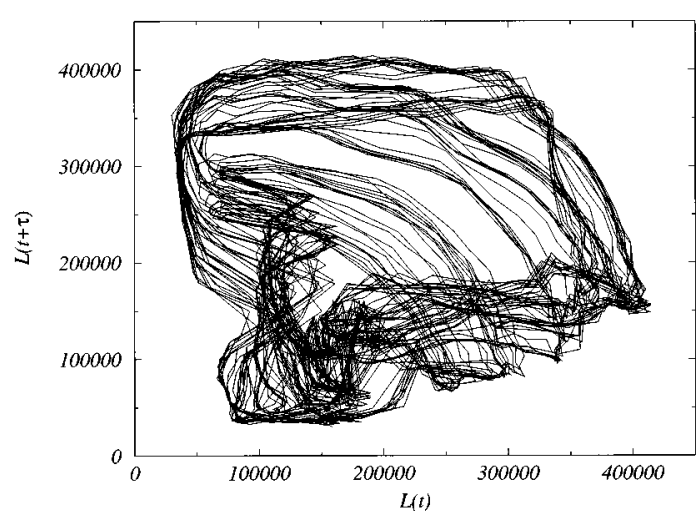

FIG. 17. $L$-induced attractor projected on the plane spanned by $\{L(t), L(t+\tau)\})$ where the time delay $\tau$ is equal to $15 \delta t$.

enough. However, every variable of a given system may not contain all the pertinent information about the dynamical behavior when one is forced to use low dimensional phase spaces, as exemplified by two of us. ${ }^{26}$ Such is usually the case when one deals with topological characterization which is limited to 3D-phase spaces. Thus, the case of the radial velocity $v_{R}$ and the luminosity $L$ must be investigated, if only as a check of our previous results.

The case of the radial velocity $v_{R}$ of the stellar surface is easy to solve since it is shown ${ }^{20}$ that derivative coordinates are equivalent to delay coordinates. The velocity $v_{R}$ being the time derivative of the stellar radius $R, v_{R}$-time series thus contains the same information about the dynamical behavior as the $R$-time series. [In the specific discrete Lagrangian hydro-code the velocity and radius are actually related by $R(t+\Delta t)=R(t)+\frac{1}{2} \Delta t\left(v_{R}(t+\Delta t)+v_{R}(t)\right)$, where $\Delta t$ is the numerical time-step, i.e., by a linear reshuffling of the variables which makes the equivalence even more straightforward.]

While the $R$ - or $v_{R}$-time series are smooth enough, the temporal behavior of the luminosity, is very jittery (Fig. 7) because Lagrangian hydrocodes have great difficulty in resolving the motion of the sharp $H$ partial ionization regions. As seen from Fig. 17, one then expects more difficulties in studying the $L$-attractor than the $R$-attractor, a fact which could possibly be improved in the future by using an adaptive hydrocode. It is therefore of particular interest to check whether the $L$-induced attractor $A_{L}$ reconstructed in a $3 \mathrm{D}$ space by a time delay method can be found to be topologically equivalent to the $R$-induced attractor $A_{R}$.

As for the attractor $A_{R}$, we begin the study by defining a Poincaré section $P_{L}$ here

$$
\begin{aligned}
& P_{L} \equiv\left\{L(t), L(t+\tau) \in \mathbb{R}^{2} \mid L(t)=1.710^{6},\right. \\
& \left.L(t+\tau)>1.510^{6}, \quad \dot{L}<0\right\}
\end{aligned}
$$

which is chosen for its computational advantages. A firstreturn map is thereafter built and displayed in Fig. 18.

Periodic orbits are extracted and encoded according to a symbolic dynamics which is given by 


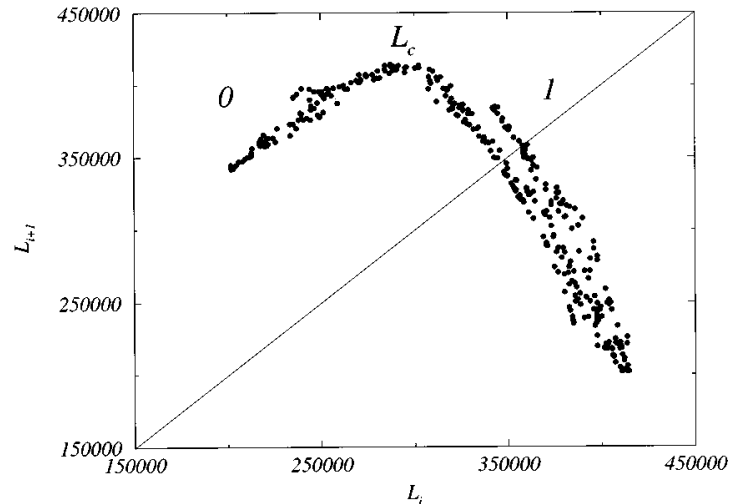

FIG. 18. First-return map to the Poincare section $P_{L}$. A critical point $L_{c}=291000$ splits the map in two branches.

$$
\begin{aligned}
& 0 \text { if } L<L_{c}, \\
& 1 \text { if } L>L_{c} .
\end{aligned}
$$

The population of periodic orbits is reported in Table I and is found to be exactly the same as the one obtained within the $R$-induced attractor. In the computation of the symbolic plane associated with the $L$-induced attractor (Fig. 19), the pruning front is better approximated by a line than in the case of the $R$-induced attractor although the $L$-attractor is more jittery than the $R$-attractor. This fact actually confirms the sensitivity of such an analysis to the initial conditions of time series when short time series are used. Consequently, it is here confirmed that the dynamics of the $\mathrm{W}$ Vir model is governed by an unimodal forcing order for the studied control parameter. The pruning front is located at $\alpha_{p}=0.8499$ which is again a little bit greater than the leading symbolic coordinate $\alpha_{(10110)}$. From the orbital point of view, both attractors $A_{R}$ and $A_{L}$ therefore contain the same information.

We have now to check whether the $L$-induced attractor possesses the same topology as the $R$-induced attractor. Due to the parasitic oscillations of the luminosity $L$, we cannot expect to correctly count oriented crossings between a pair of

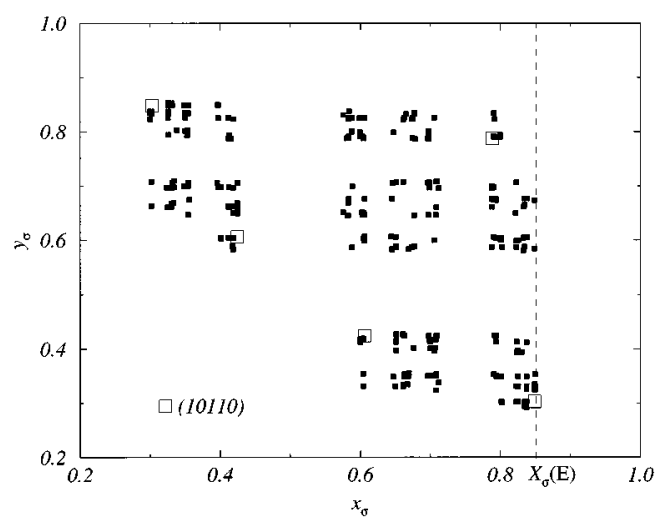

FIG. 19. Symbolic plane associated with the $L$-induced attractor. The pruning front is well approximated by the dashed line which is located at $\alpha_{p}=0.8499$. The period-5 orbit encoded by (10110) is reported on this plane.

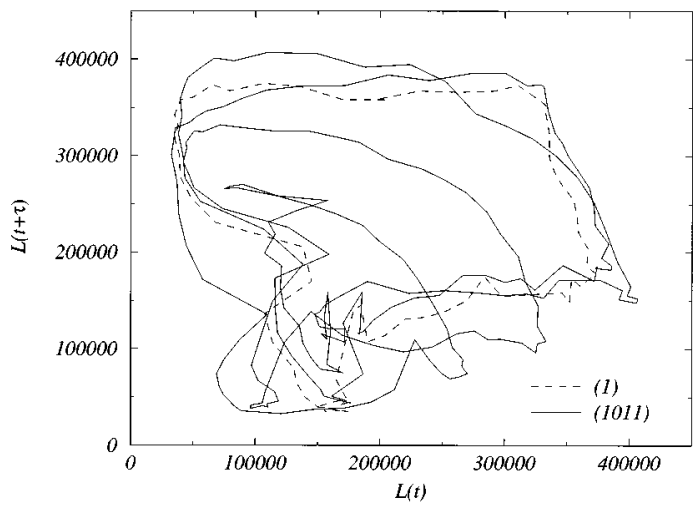

FIG. 20. The small sampling rate and the presence of many erratic oscillations prevent a correct counting of oriented crossings.

orbits when one period in the pair is large (greater than or equal to typically 4). For instance, a plane projection of the pair $(1011,1)$ is displayed in Fig. 20. The small sampling rate and the presence of many erratic oscillations prevent the determination of the linking number $L(1011,1)$.

Consequently, only the pair $(10,1)$ can be used to compute a linking number since period-3 orbits are not present within the attractor. The linking number $L(10,1)$ predicted by the $R$-induced template is

$$
\begin{aligned}
L(10,1) & =\frac{1}{2}\left[M(1,1)+M(1,0)+N_{\text {lay }}(10,1)\right] \\
& =\frac{1}{2}[+1+0+1]=+1,
\end{aligned}
$$

where the layering number $N_{\text {lay }}(10,1)$ is given by the layering graph displayed in Fig. 21.

With the help of a plane projection of the pair $(10,1)$ the linking number $L(10,1)$ is found to be equal to +1 in agreement with the $R$-template prediction (see Fig. 22).

In order to try to compute linking numbers with orbits of higher periods, we applied a smoothing to the data. Many oscillations are removed by the filtering process. Nevertheless, the linking number $L(10,1)$ counted on a plane projection of orbit (10) and (1) extracted from the smoothed $L$-time series is now found to be equal to -1 (Fig. 23). The filtering process therefore does not preserve the topology of the original data and cannot be safely used. It is then difficult to provide a definitive conclusion concerning the topology of the $L$-induced attractor. However, we may state that, relying on the obtained results, the topology of the $L$-induced attractor is compatible with the topology of the $R$-induced attrac-

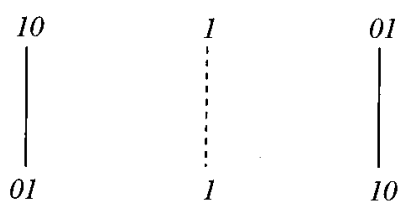

FIG. 21. The layering number $N_{\text {lay }}(10,1)$ is found to be equal to +1 . 


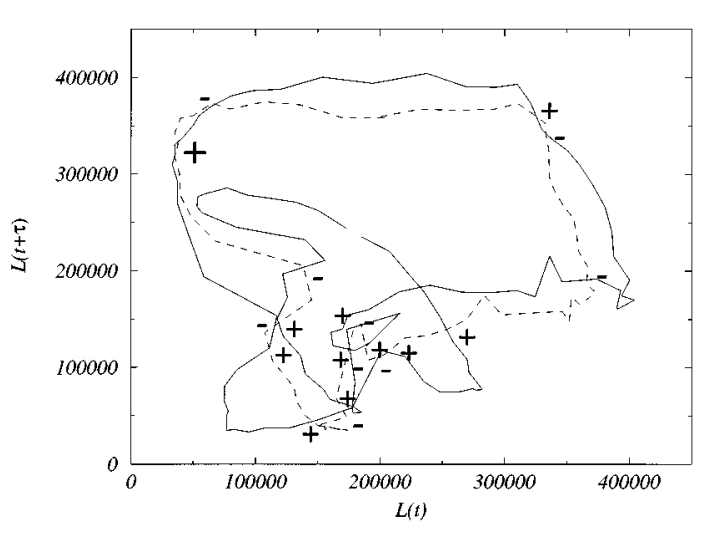

FIG. 22. Plane projection of the pair $(10,1): L(10,1)=\frac{1}{2}(-9+11)=+1$.

tor. If we furthermore recall that the populations of periodic orbits have been found to be the same for the $R$ - and for the $L$-induced attractors, we may conclude that both attractors are, at least, very close and, very likely, equivalent.

\section{CONCLUSION}

In this paper we have applied the topological analysis, a novel tool in astrophysics, to the analysis of the W Vir model pulsations that were generated by a numerical hydrodynamical code. The banded W Vir attractor that is reconstructed from the temporal behavior of the stellar radius is found to possess an additional $\pi$-twist when compared to the Rössler band. Since the topological properties of the dynamics that are deduced from the model must be in agreement with observational data, this then provides a novel type of constraint on the stellar models and a check on the physics that is included in the hydrocode. For instance, if the twist is indeed a robust property of $\mathrm{W}$ Vir type stars, it should then also be uncovered in observed light-curve data when they become available. However at the present time we do not have a good understanding of the physical nature of this additional $\pi$-twist, and how it comes about in this oscillating system.

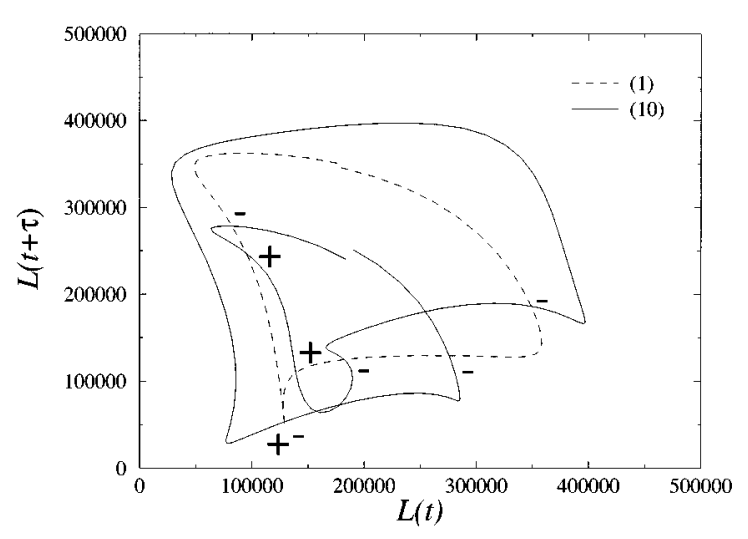

FIG. 23. Plane projection of the periodic orbits encoded by (10) and (1), respectively. They are extracted from the smoothed luminosity time series. The linking number is found to be different from the one counted on the nonfiltered data.
The attractors obtained from the stellar radius and from the luminosity time series possess the same population of periodic orbits. Due to the jittery character of the luminosity time series, the topology of the $L$-induced attractor cannot be characterized completely safely, though. Nevertheless, our results show that the topologies of the $R$ - and of the $L$-induced attractors are found to be compatible, and in fact it is likely that both attractors are actually equivalent. The stellar light-curve is the most accurate and often the only observable quantity. From a practical point of view it is therefore reassuring to the astrophysicist that the luminosity is generic in the sense that it contains the same dynamical information as the temporal variations of the surface radius or the surface radial velocity. It can thus safely be used to infer the physical and mathematical properties of this dynamics.

Finally we note that unfortunately, at the present time, the topological characterization is only available for 3D systems whose dynamics is very dissipative. Until an extension to higher dimensions can be found our method therefore cannot be applied to more complicated observational stellar light-curves, such as R Scuti, which have a fractal dimension greater than $3 .^{27}$

\section{ACKNOWLEDGMENTS}

This research has been supported in part by the National Science Foundation (Grants No. AST 92-18068 and No. INT94-15868).

${ }^{1}$ J. R. Buchler and G. Kovács, "Period doubling bifurcations and chaos in W Virginis models," Astrophy. J. Lett. 320, L57-L62 (1987).

${ }^{2}$ G. Kovács and J. R. Buchler, "Regular and irregular nonlinear pulsations in population II Cepheid models," Astrophys. J. 334, 971-994 (1988).

${ }^{3}$ J. R. Buchler, "Chaotic pulsations in stellar models," Ann. N.Y. Acad. Sci. 617, 17-36 (1990).

${ }^{4}$ N. H. Packard, J. P. Crutchfield, J. D. Farmer, and R. S. Shaw, “Geometry from a time series," Phys. Rev. Lett. 45, 712-716 (1980). The ideas here discussed were also put forward by Ruelle and Takens (not published).

${ }^{5}$ G. B. Mindlin, X. J. Hou, H. G. Solari, R. Gilmore, and N. B. Tufillaro, "Classification of strange attractors by integers," Phys. Rev. Lett. 64, 2350-2353 (1990).

${ }^{6}$ N. B. Tufillaro, T. Abbott, and J. Reilly, An Experimental Approach to Nonlinear Dynamics and Chaos (Addison-Wesley, New York, 1992).

${ }^{7}$ O. E. Rössler, "An equation for continuous chaos," Phys. Lett. A 57, 397-398 (1976).

${ }^{8}$ J. S. Birman and R. F. Williams, "Knotted periodic orbits in dynamical systems: Lorenz's equations," Topology 22, 47-82 (1983).

${ }^{9}$ P. Melvin and N. B. Tufillaro, "Templates and framed braids," Phys. Rev. A 44, 3419-3422 (1991).

${ }^{10}$ C. Letellier, P. Dutertre, and B. Maheu, "Unstable periodic orbits and templates of the Rössler system: Toward a systematic topological characterization," Chaos 5, 271-282 (1995).

${ }^{11} \mathrm{P}$. Collet and J. P. Eckmann, Iterated Maps on the Interval as Dynamical Systems, Progress in Physics, edited by Jaffe and D. Ruelle (Birkhäuser, Boston, 1980).

${ }^{12}$ T. Hall, "The creation of horseshoes," Nonlinearity 7, 861-924 (1994).

${ }^{13}$ N. B. Tufillaro, P. Wyckoff, R. Brown, T. Schreiber, and T. Molteno, "Topological time series analysis of a string experiment and its synchronized model,"' Phys. Rev. E 51, 164-174 (1995).

${ }^{14}$ H. P. Fang, "Dynamics of strongly dissipative systems," Phys. Rev. E 49, 5025-5031 (1994).

${ }^{15}$ P. Cvitanović, M. Gunaratne, and I. Procaccia, "Topological and metric properties of Héon-type map strange attractors," Phys. Rev. A 38, 15031520 (1988).

${ }^{16} \mathrm{~J}$. Milnor and W. Thurston, "On iterated maps of the interval," Lect. Notes Math. 1342, 465-563 (1988). 
${ }^{17}$ L. Le Sceller, C. Letellier, and G. Gouesbet, "Algebraic evaluation of linking numbers of unstable periodic orbits in chaotic attractors," Phys. Rev. E 49, 4693-4695 (1994).

${ }^{18}$ G. B. Mindlin, H. G. Solari, M. A. Natiello, R. Gilmore, and X. J. Hou, "Topological analysis of chaotic time series data from the BelousovZhabotinski reaction," J. Nonlinear Sci. 1, 147-173 (1991).

${ }^{19}$ T. Serre, Z. Kolláth, and J. R. Buchler, "Search for low dimensional chaos in variable stars-the global polynomial phase space reconstruction method," Astron. Astrophys. 311, 833-844 (1986); ibid. 311, 845-851 (1986).

${ }^{20}$ J. F. Gibson, J. D. Farmer, M. Casdagli, and S. Eubank, "An analytic approach to practical state space reconstruction," Physica D 57, 1-30 (1992).

${ }^{21}$ P. Grassberger, H. Kantz, and U. Moening, "'On the symbolic dynamics of the Hénon map,' J. Phys. A 22, 5217-5230 (1989).
${ }^{22}$ H. P. Fang, "Universal bifurcation property of two- or higher-dimensional dissipative systems in parameter space: Why does 1D symbolic dynamics work so well?,' J. Phys. A 28, 3901-3910 (1995).

${ }^{23} \mathrm{P}$. Dutertre, Caractérisation des attracteurs étranges par leurs populations d'orbites périodiques, Thème cycle, LESP, URA CNRS 230, CORIA, Rouen, 1995.

${ }^{24}$ P. Dutertre, C. Letellier, and G. Gouesbet, "Evolution of multimodal maps induced by 3D flows. I. The case of the Rössler system"' (in preparation)

${ }^{25}$ E. Ott, Chaos in Dynamical Systems (Cambridge University Press, Cambridge, 1993).

${ }^{26}$ C. Letellier and G. Gouesbet, "Equivalences between original and reconstructed strange attractors", (in preparation).

${ }^{27}$ J. R. Buchler, T. Serre, Z. Kolláth, and J. Mattei, Phys. Rev. Lett. 74, 842-845 (1995) 